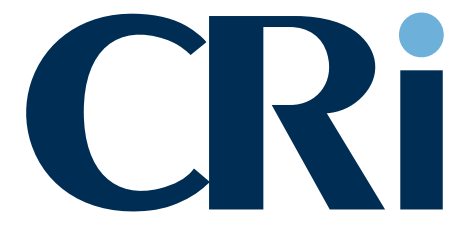

Issue 6

15 December 2006

P. $161-192$

PVSt 52028
A Journal of Information Law and Technology

\title{
Articles
}

Frank R. Remmertz - Alternative Dispute Resolution (ADR) an alternative for eu domain name disputes?

Henrik Nilsson - Different Approaches in Nordic File Sharing Case Law

Anja E. Dekhuijzen - Five Risk Factors in Outsourcing Agreements

Probir Roy Chowdhury - Use of RFID Technology

\section{Case Law}

USA: Liability of Interactive Computer Services for User Postings Chicago Lawyer's Committee for Civil Rights under the Law, Inc. v. Craigslist, Inc.

UK: Liability of ISP for Defamatory Posting John Bunt v. David Tilley et al.

\section{Updates}

Peter Blume - Denmark: Data Retention

Guillaume Le Lu - France: Copyright Law and DRMs

loannis Iglezakis - Greece: Re-Use of Public Sector Information

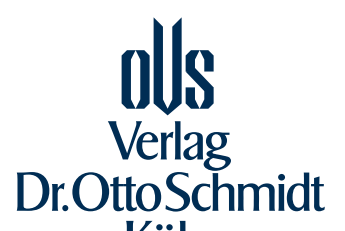

Köln 


\section{Einfach klasse!}

\section{Besser kann man das}

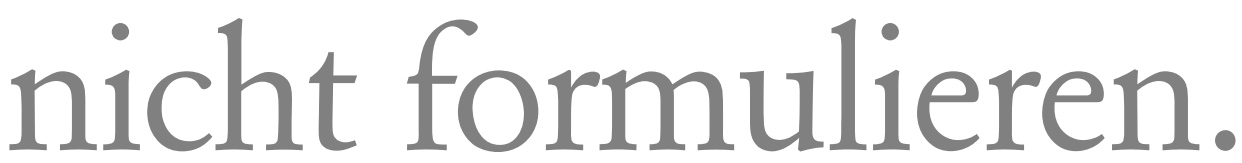

Das Rechtsformularbuch. Die neue Auflage dieses Klassikers für mustergültige Rechtsgestaltung gibt Ihnen über 1.000 bewährte, vielfach erprobte Muster und Formulare auf aktuellem Stand an die Hand. Praxisgerecht in einem Band.

Einführende, musterbezogene Erläuterungen in jedem Kapitel helfen Ihnen, sich auch in Rechtsgebieten schnell zurechtzufinden, die Ihnen nicht so geläufig sind, und ermöglichen Ihnen in jedem Fall die perfekte Anpassung eines Musters an den konkreten Regelungssachverhalt.

Die Autoren, eine gelungene Mischung erfahrener und versierter Praktiker aus allen relevanten Berufen, unterstützen Sie mit wertvollen

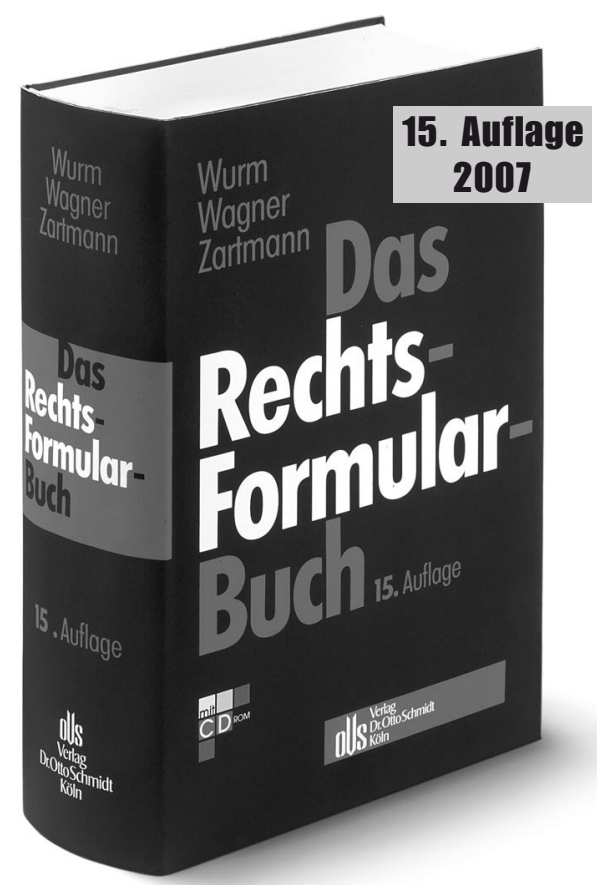

Wurm/Wagner/Zartmann Das Rechtsformularbuch 15., völlig neu bearbeitete Auflage 2007, 2.537 Seiten Lexikonformat, gbd., inkl. CD mit den Mustern 129,- $€[D]$.
Praxistipps und hilfreichen Checklisten. Es gibt zahlreiche Kostenanmerkungen und Hinweise auf steuerliche Konsequenzen. Kurzum mit dem Rechtsformularbuch können alle arbeiten: Rechtsanwälte, Notare, Unternehmensjuristen, Steuerberater, Wirtschaftsprüfer.

Über die mitgelieferte CD stehen Ihnen die Muster und Formulare natürlich auch für den individuellen Einsatz am PC zur Verfügung. Einfach, schnell und komfortabel können sie verändert, bearbeitet und in Ihre eigenen Dateien übernommen werden.

Das Rechtsformularbuch. Einfach klasse! Wie wär's mit einer Leseprobe? www.otto-schmidt.de

Bestellschein ausfüllen und faxen (02 21) 937 38-943 조옹

X Ja, ich bestelle mit 14-tägigem Rückgaberecht Wurm/Wagner/Zartmann Das Rechtsformularbuch 15. Auflage, inkl. CD mit den Mustern 129,- € [D]. ISBN 10: 3-504-07008-0 · ISBN 13: 978-3-504-07008-3

\begin{tabular}{lcccc}
\hline Name & Straße & PLZ & \\
& & & \\
\hline Telefon & Fax & Datum Unterschrift
\end{tabular}


New at www.cr-international.com: International Privacy Survey by Privacy International of 2 November 2006

\section{Computer Law Review International}

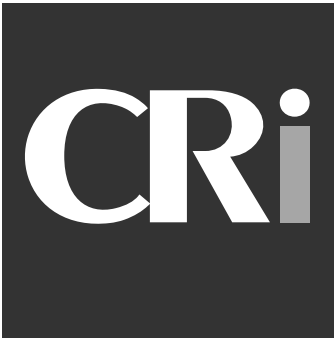

A Journal of Information Law and Technology

\section{Editorial Board}

Prof. Dr. Thomas Dreier, M.C.J.

University of Karlsruhe

Dr. Jens-L. Gaster, principal administrator, Brussels

RA Thomas Heymann, Frankfurt/M.

Prof. Dr. Michael Lehmann, Dipl.-Kfm

Max-Planck-Institute and University of Munich

Prof. Raymond T. Nimmer, University of Houston

Attorney at Law Holly K. Towle, J.D., Seattle

Attorney at Law Thomas Vinje, Brussels

\section{Articles}

Frank R. Remmertz - Alternative Dispute Resolution (ADR) - an alternative for .eu domain name disputes?

Henrik Nilsson - Different Approaches in Nordic File Sharing Case Law

Anja E. Dekhuijzen - Five Risk Factors in Outsourcing Agreements Recent international case law on failed outsourcing

Probir Roy Chowdhury - Use of RFID Technology 173

\section{Case Law}

Attorney at Law Sakari Aalto (Finland)

Attorney at Law Jonathan Band (USA)

Prof. Dr. Janusz Barta (Poland)

Abogado Enrique J. Batalla (Spain)

John P. Beardwood (Canada)

Prof. Dr. Jon Bing (Nomay)

DDr. Walter Blocher (Austria)

Prof. Peter Blume (Denmark)

Awocato Gabriel Cuonzo (Italy)

Dr. Jens-L. Gaster (EU)

Prof Ysolde Gendreau (Canada)

Dr. Lucie Guibault (Canada/Netherlands)

Avocat Dr. Martin Hauser (France)

Prof. Dr. Rosa Julia-Barcelo (Spain)

Attorney at Law Charles H. Kennedy (USA)

Dr. Stanley Lai (Singapore)

Prof. Ian Lloyd (UK)

RA Prof. Dr. Michail Marinos (Greece)

Prof. Dr. Ryszard Markiewicz (Poland)

Antonio Millé (Argentina)

Ken Moon (New Zealand)

Prof. Raymond T. Nimmer (USA)

Advogado Manuel Oehen Mendes (Portugal)

Prof. Jerome Reichman (USA)

Luis C. Schmidt (Mexico)

Harry Small (UK)

Prof. Alain Strowel (Belgium)

Awocato Pietro Tamburrini (ltaly)

Attorney at Law Thomas Vinje (USA, EU)

Prof. Coenraad J. Visser (South Africa)

Prof. Dr. Rolf H. Weber (Switzerland)

J.T. Westermeier (USA)

Neil J. Wilkof (Israel)

Jamie Wodetzki (Australia)
USA: Liability of Interactive Computer Services for User Postings Chicago Lawyer's Committee for Civil Rights under the Law, Inc. v. Craigslist, Inc. District Court for the Northern District of Illinois Eastern Division - Decision of 14 November 2006

USA: GPL And Anti-Trust Law Danie/ Wallace v. IBM Corp., Red Hat, Inc. and Novell, Inc. - Court of Appeals for the 7th Circuit - Decision of 9 November 2006

USA: Damages For Blacklisting Spamming Companies e360 Insight, LLC et al. v. The Spamhouse Project - District Court for the Northern District of IIIInois - Decision of 13 September 2006

UK: Liability of ISP for Defamatory Posting John Bunt v. David Tilley et al. - High Court of Justice Decision of 10 March 2006

USA: Personal Jurisdiction Over Non-Residents Yahoo! Inc. v. La Ligue Le Racisme et Antisemitisme - Court of Appeals for the 9th Circuit - Decision of 12 January 2006

\section{Updates}

Peter Blume - Denmark: Data Retention

Guillaume Le Lu - France: Copyright Law and DRMs

loannis Iglezakis - Greece: Re-Use of Public Sector Information

- About the Authors

- Masthead

- CRI-Reader Services 


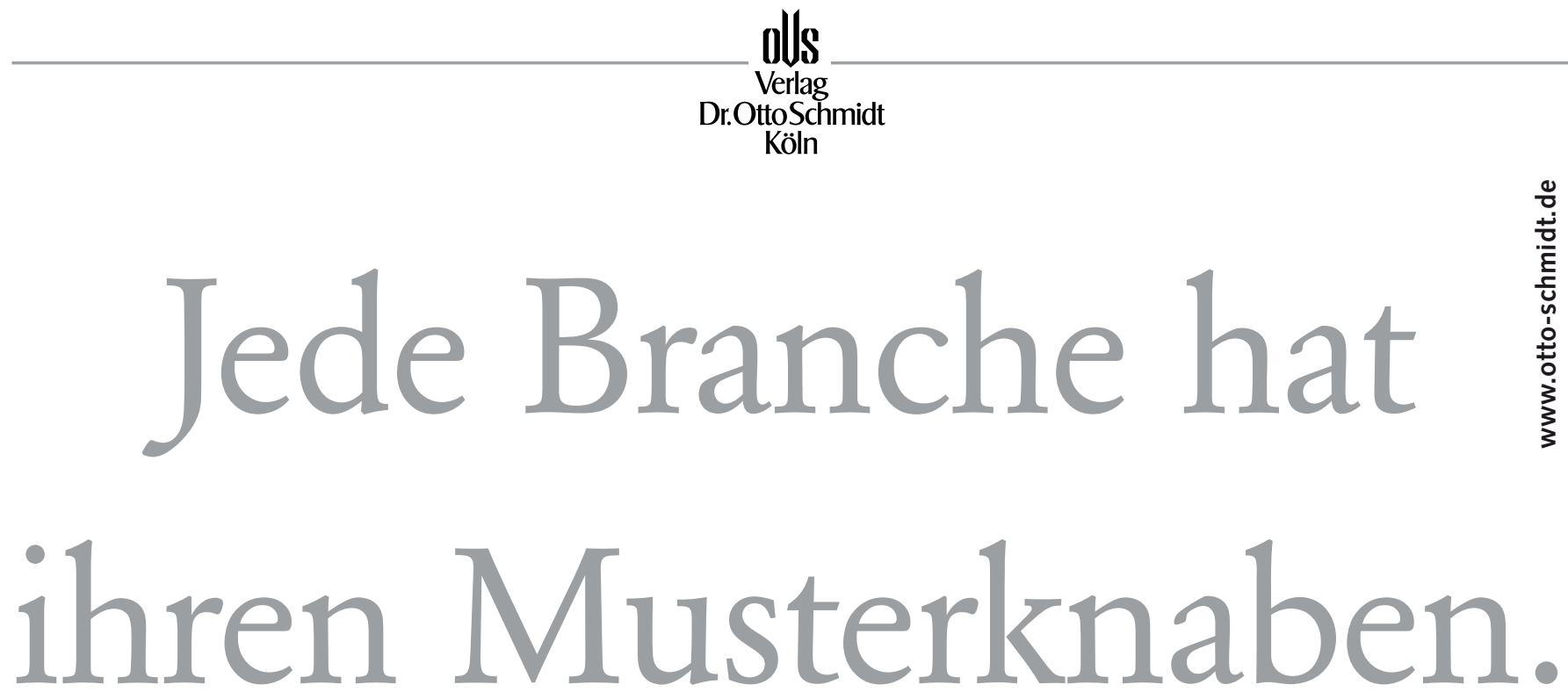

Es ist das erste Handbuch, das ITVerträge kommentiert. Klausel für Klausel. Absatz für Absatz. Damit Sie die komplizierten Sachverhalte der Materie in jedem Einzelfall durchschauen und so beim Abschluss von Verträgen zu besseren Ergebnissen kommen.

Das Handbuch deckt mit seinen Vertragsmustern alle modernen Kommunikationsbereiche ab. Sprich EDV-, IT- und TK-Recht. Auf unzulässige Klauseln werden Sie hingewiesen, für Besonderheiten und abweichende Interessenlagen bietet man Ihnen alternative Formulierungen an. Und alle Vertragsmuster finden Sie auch auf der mitgelieferten CD für die praktische Arbeit am PC.

Die einzelnen Vertragstypen spie-

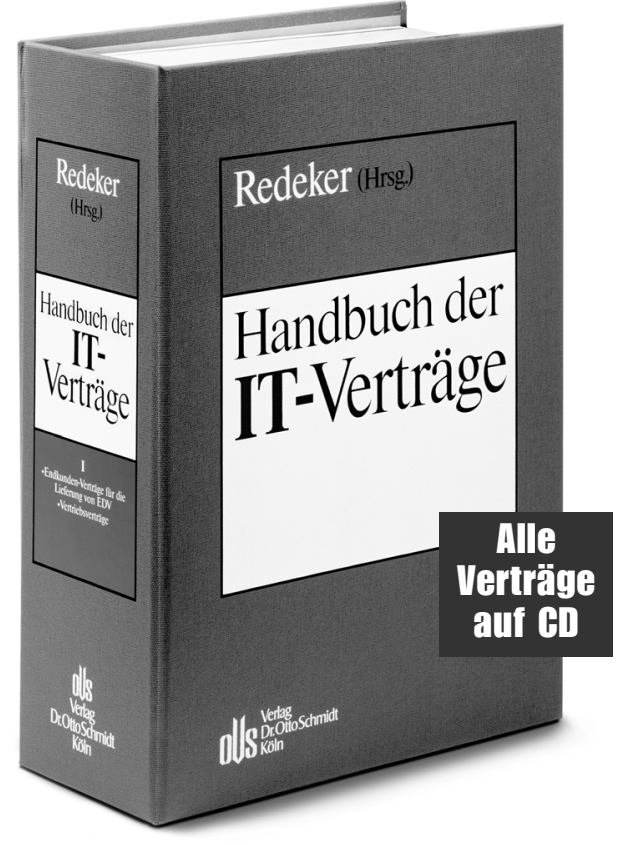

Redeker (Hrsg.) Handbuch der IT-Verträge Loseblatt, z.Zt. 2.774 Seiten in 2 Ordnern, inkl. CD mit allen Mustern. Nur 159,- $€[$ [D] mit Abo-Verpflichtung für mindestens zwe Jahre. Ergänzungslieferungen/Updates 1-3-mal im Jahr. ISBN 3-504-56008-8. Ohne Abo 299,- $€[$ [D]. ISBN 3-504-56027-4 geln den aktuellen Stand der Praxis im IT-Recht wider und werden weiter um neue praxisrelevante Muster ergänzt. Die Autoren sind sehr erfahrene Praktiker - Rechtsanwälte, die solche Verträge oft erarbeitet, verhandelt und erfolgreich abgewickelt haben. Sie geben ihr gesamtes Know-how in diesem Handbuch an Sie weiter und halten Sie in dem dynamischen Rechtsgebiet auf dem Laufenden. Leseprobe? www.otto-schmidt.de

Am besten sehen Sie sich in aller Ruhe an, was in dem Handbuch der IT-Verträge drinsteckt. 14 Tage kostenlos und unverbindlich. Bestellen Sie Ihr persönliches Probeexemplar. Und Sie werden sehen: So gut ging Ihnen die Vertragsgestaltung noch nie von der Hand.

Bestellschein ausfüllen und faxen (02 21) 937 38-943

Ja, ich bestelle mit 14-tägigem Rückgaberecht Redeker (Hrsg.) Handbuch der IT-Verträge Loseblatt, z.Zt. 2.774 Seiten in 2 Ordnern, inkl. CD mit allen Mustern. (Zutreffendes bitte ankreuzen!)

nur 159,- $€[$ [D] mit Abo-Verpflichtung für mindestens zwei Jahre. Ergänzungslieferungen/Updates bis auf Widerruf 1-3-mal im Jahr. ISBN 3-504-56008-8

ohne Abo-Verpflichtung 299,- $€[D]$. ISBN 3-504-56027-4 